\title{
Dinâmica da infecção de Babesia bovis (Babés, 1888, Starcovici, 1893) em fêmeas ingurgitadas e ovos de Boophilus microplus (Canestrini, 1887)
}

\author{
Dynamic of infection of Babesia bovis (Babés, 1888, Starcovici, 1893) in engorged females \\ and eggs of Boophilus microplus (Canestrini, 1887)
}

\section{Jairo Dias Barreira ${ }^{1}$ Maria Inês Doria Rossi ${ }^{2}$ Fabiano Araujo Pires ${ }^{3}$ Gil Vicente Oliveira da Silva ${ }^{3}$ Carlos Luiz Massard $^{3}$}

\section{RESUMO}

\begin{abstract}
A dinâmica da infecção de B. bovis no carrapatovetor B. microplus foi estudada em condições laboratoriais na Universidade Federal Rural do Rio de Janeiro no Laboratório de Protozoologia. Para tanto, foram examinadas 100 fêmeas ingurgitadas que se desprenderam naturalmente do hospedeiro vertebrado, sendo que 84 fêmeas apresentaramse infectadas com esporocinetos de $\boldsymbol{B}$. bovis, com a seguinte distribuição: $39 \%$, 33\%, e 12\% nos dias 3,4 e 5 de incubação, respectivamente. Foram obtidas amostras de ovos provenientes das fêmeas positivas para B. bovis, $100 \%$ das amostras de ovos estavam infectadas, apresentado a seguinte distribuição: 46,4\%, 34,5\%, 16,7\% e 2,4\% nos dias 4, 5, 6 e 7 de incubação, respectivamente. As freqüencias acumuladas, tanto de fêmeas infectadas (84\%) quanto de ovos infectados (100\%) mantiveram-se até o 17o dia de incubação. De acordo com as freqüencias acumuladas e o aumento do grau de infecção, conclui-se que amostras coletadas a partir do $5^{\circ} e$ 7 o dia de incubação, são ideais para o diagnóstico de $\boldsymbol{B}$. bovis, em hemolinfa e ovos, respectivamente.
\end{abstract}

Palavras-chave: infeç̧ão, B. bovis, B. microplus.

\section{ABSTRACT}

The dynamics of $\boldsymbol{B}$. bovis infections in the tick-vector B. microplus, was studied in laboratory conditions at Universidade Federal Rural do Rio de Janeiro in the Laboratory of Protozoology, Brazil.. To do it, 100 engorged females naturally droped from vertebrate host were examined. From this total 84 females showed infected with sporokinets of $\boldsymbol{B}$. bovis, with the following distribution: $39 \%, 33 \%$ and $12 \%$ on days 3,4 and 5 of incubation, respectivelly. Eggs samples were obtained from the positives ones to B. bovis females and $100 \%$ were infected, showed the follow distribution: $46.4 \%, 34.5 \%, 16.7 \%$ and $2.4 \%$ on days $4,5,6$ and 7 of incubation, respectivelly. The accumulative frequencies, of infected females (84\%) and infected eggs (100\%), were kept until $17^{\text {th }}$ days of incubation. According to the accumulatives frequencies and the increase of the degree of incubation, it was concluded that samples collect on days $5^{\text {th }}$ to $7^{\text {th }}$ of incubation, are the best for diagnosis of $\boldsymbol{B}$. bovis in hemolymph and eggs, respectively.

Key words: infection, B. bovis, B. microplus.

\section{INTRODUÇÃO}

O envolvimento dos carrapatos na transmissão de protozoário do gênero Babesia foi descrito pela primeira vez por SMITH \& KILBORNE (1893) ao relacionar o surto de babesiose observado em bovinos no sul dos Estados Unidos com a presença de Boophilus annulatus. Estudos caracterizando a presença e desenvolvimento de Babesia spp. no intestino, hemolinfa e ovário foram demostrados por KOCH (1906), CRAWLEY (1915) e ROSENBUSCH (1927) nos vetores Rhipicephalus evertsi, B. annulatus, B. microplus, respectivamente. Em condições experimentais, RIEK(1964, 1966) observou o comportamento,

${ }^{1}$ Programa de Pós-graduação em Ciências Veterinárias, Parasitologia Veterinária. E-mail: jairobarreira@bol.com.br. Rua Venâncio Veloso, 35, Ap.102, 22790-420, Recreio dos Bandeirantes, Rio de Janeiro, RJ, Brasil.

${ }^{2}$ Departamento de Fisiologia e Farmacodinâmica Fundação Instituto Osvaldo Cruz, RJ, Brasil.

${ }^{3}$ Departamento de Parasitologia Animal Instituto de Veterinária, Universidade Federal Rural do Rio de Janeiro, RJ, Brasil. 
tempo de evolução e diferentes fases evolutivas de $\boldsymbol{B}$. bigemina e $\boldsymbol{B}$. bovis nos vários órgãos de fêmeas ingurgitadas, ovos, larvas e ninfas de $\boldsymbol{B}$. microplus. Nos trabalhos desenvolvidos por AKINBOADE \& DIPEOLU (1981), GUGLIELMONE et al (1985, 1996 e 1997), BARREIRA (1988 e 2001), MELENDEZ \& FORLANO (1996), foram observados diferenças significativas no comportamento dos esporocinetos de $\boldsymbol{B}$. bovis e $\boldsymbol{B}$. bigemina nos diferentes órgãos, bem como nos parâmetros biológicos, taxa, grau e tempo de infecção no hospedeiro vertebrado. A necessidade de conhecer estes parâmetros para estabelecer de forma precisa a dinâmica da infecção, especificamente $\boldsymbol{B}$. bovis, no carrapato vetor $B$. microplus, constituiu o objetivo deste trabalho.

\section{MATERIAL E MÉTODOS}

Para a realização do experimento, foram utilizados três animais (BM1, BM2 e BM3) mestiços (Bos taurus $X$ B. indicus), machos, não esplenectomizados, com seis meses de idade, originados do Planalto do Itatiaia, a $22^{\circ} 44^{\prime}$ de latitude sul e $44^{\circ} 60^{\prime}$ de longitude oeste, a 2400 metros de altitude, região livre de $\boldsymbol{B}$. bovis e do vetor $\boldsymbol{B}$. microplus. Após avaliação clínica, hematológica e parasitológica, os animais foram mantidos em baias individuais sob condições de isolamento, localizadas na Estação para Pesquisas Parasitológicas W.O. Neitz do Departamento de Parasitologia Animal, Instituto de Veterinária, UFRRJ.

Obtenção de larvas de $\boldsymbol{B}$. microplus livres de infecção por Babesia spp

Fêmeas ingurgitadas de $\boldsymbol{B}$. microplus foram obtidas de bovinos naturalmente infestados, mantidos no Instituto de Zootecnia da Universidade Federal Rural do Rio de Janeiro. No Laboratório de Protozoologia, as fêmeas foram distribuídas em placas de Petri previamente identificadas e mantidas em condições laboratoriais (incubadas em câmaras climatizada à temperatura de $28^{\circ} \mathrm{C}$ e umidade relativa superior a $80 \%$ ). Ovos do primeiro e segundo dia de postura foram colhidos, pesados em balança analítica, separados em amostras de $0,2 \mathrm{~g}$ e acondicionados para criação e manutenção de carrapatos (NEITZ et al., 1971; BENNETT, 1974). Para evidenciar uma possível infecção por $\boldsymbol{B}$. bovis, em ovos até o segundo dia de ovipostura, as amostras foram separadas, coradas pelo Giemsa e examinadas com o auxílio de microscopia óptica. Dez dias após a eclosão, as larvas foram utilizadas para infestação de um hospedeiro sensível (BM1), permitindo comprovar a negatividade das larvas de carrapatos utilizadas durante o experimento.

Obtenção de larvas de $\boldsymbol{B}$. microplus infectadas com B.bovis

Para obtenção das larvas infectadas, foi utilizado um bezerro (BM2) infestado durante 12 dias consecutivos com aproximadamente 4.000 larvas de B. microplus livres de Babesia spp, oriundas de 0,2g de ovos de início de postura conforme a técnica utilizada por THOMPSON (1976), MAHONEY \& MIRRE (1977) e DE VÓS et al. (1984), modificada.

Quinze dias após a primeira infestação, o bezerro foi inoculado com $20 \mathrm{ml}$ de sangue bovino infectado com $\boldsymbol{B}$. bovis, permitindo assim, que a parasitemia provocada pela amostra de sangue coincidisse com a repleção e queda natural das fêmeas ingurgitadas originadas das infestações iniciais.

As fêmeas ingurgitadas que se desprenderam naturalmente receberam o mesmo tratamento adotado para a população obtida a campo. Posteriormente à seleção, as fêmeas ingurgitadas infectadas foram mantidas em placas de Petri e fixadas em fitas adesivas, o que permitiu, selecionar as amostras de ovos infectados. Os ovos utilizados para a obtenção de larvas de B. microplus foram selecionados no período de seis a doze dias após o início da postura, a partir de fêmeas ingurgitadas comprovadamente infectadas pela presença de esporocinetos em esfregaços de hemolinfa. Os ovos foram separados, com o auxílio de um pincel fino, em amostras de $0,5 \mathrm{~g}$ e acondicionados em seringas descartáveis para a criação e manutenção dos carrapatos. As seringas foram identificadas e os ovos incubados conforme descrito anteriormente.

Infecção experimental das fêmeas ingurgitadas de $\boldsymbol{B}$. microplus

Para obtenção das fêmeas infectadas, foi utilizado um bezerro (BM3), sensível a B. bovis infestado experimentalmente com aproximadamente 4.000 larvas de $\boldsymbol{B}$. microplus não infectadas durante 12 dias consecutivos. Após a sexta infestação, o hospedeiro foi submetido ao desafio com aproximadamente 10.000 larvas de $\boldsymbol{B}$. microplus infectadas $\operatorname{com} \boldsymbol{B}$. bovis. As fêmeas ingurgitadas, após desprenderem-se naturalmente do hospedeiro, foram coletadas, levadas ao laboratório e mantidas sob as mesmas condições de temperatura e umidade aplicadas aos grupos anteriores. Estas fêmeas foram coletadas no período em que a parasitemia observada foi de $0,4 \%$ para B. bovis.Para estudar a dinâmica da infecção de

Ciência Rural, v. 35, n. 5, set-out, 2005. 
B. bovis no carrapato B. microplus, 100 fêmeas ingurgitadas, previamente identificadas foram examinadas a partir de amostras de hemolinfa até o $17^{\circ}$ dia de incubação, período no qual as fêmeas permaneceram viáveis. Para a obtenção, preparo e exame das amostras de hemolinfa foi aplicada a metodologia descrita por BURGDORFER (1970), BARREIRA (1988 e 2001). Os esfregaços de hemolinfa foram preparados a partir da secção do tarso e tíbia com auxílio de tesoura para microcirurgia. As amostras de hemolinfa foram depositadas em lâminas de vidro para microscopia, secadas em temperatura ambiente, fixadas em metanol por três minutos e coradas pelo Giemsa. Após a coloração, as lâminas foram examinadas em microscopia óptica, com aumento de 1.000 vezes.

As amostras de ovos utilizadas durante esta fase experimental, foram obtidas do grupo de fêmeas ingurgitadas comprovadamente infectadas (84 fêmeas) com B. bovis, selecionadas para o estudo da dinâmica da infecção. As amostras de ovos foram coletadas diariamente, durante o período compreendido entre o primeiro e o 17o dia de postura. Os ovos foram macerados, fixados em metanol e corados pelo Giemsa.

Cálculo do grau de infecção das fêmeas ingurgitadas Para calcular o grau de infecção das fêmeas ingurgitadas, foi utilizado como referência básica o trabalho de FRIEDHOFF \& SMITH (1981). As amostras de hemolinfa coletadas para o estudo das formas evolutivas de $\boldsymbol{B}$. bovis, também, foram utilizadas para o cálculo do grau de infecção das fêmeas ingurgitadas de $\boldsymbol{B}$. microplus. Os exames das amostras foram realizados diariamente, utilizando-se a microscopia óptica, com magnitude de $1000 \mathrm{x}$. obedecendo ao seguinte critério: grau 1, 1 a 5 esporocinetos por campo microscópico; grau 2, 6 a 50 esporocinetos por campo; grau 3, 51 a 100 esporocinetos por campo e grau 4, acima de 100 esporocinetos por campo.

\section{RESULTADOS E DISCUSSÃO}

Do total de 100 fêmeas ingurgitadas examinadas após a queda natural do hospedeiro BM3, 84 fêmeas apresentaram-se infectadas por B. bovis, sendo que $39 \%$ no terceiro dia, $33 \%$ no quarto dia e $12 \%$ no quinto dia de incubação. A freqüência acumulada de $84 \%$ das fêmeas ingurgitadas infectadas alcançadas no quinto dia permaneceu até o décimo sétimo dia de incubação. Entretanto, um grupo de fêmeas ingurgitadas coletadas no mesmo hospedeiro, com o mesmo grau de parasitemia (0,4\% para B.bovis) utilizado para obtenção de larvas, permaneceu infectado até $21^{\circ}$ dia de incubação, porém com um número reduzido de esporocinetos.

Durante o período de incubação, as fêmeas ingurgitadas positivas para $\boldsymbol{B}$. bovis apresentaram diferentes graus de infecção (grau 1, 2, 3 e 4). O grau 1 foi observado após terceiro dia, permanecendo até o sétimo dia de incubação. O pico máximo foi registrado no quarto dia, quando 85,71\% das fêmeas ingurgitadas apresentaram-se infectadas com um a cinco esporocinetos por campo. O grau 2 foi calculado através das amostras examinadas entre o quinto e o 13ํ dia de incubação. Nestas amostras, o pico máximo foi obtido no sexto dia, quando $91,66 \%$ das fêmeas ingurgitadas apresentaram-se com 6 a 50 esporocinetos por esfregaço. O início do grau 3 ocorreu no sexto dia, persistindo até o décimo sétimo dia. O pico máximo foi observado no $11^{\circ}$ dia, em 98,8\% das fêmeas ingurgitadas, embora as amostras examinadas entre 0 oitavo e $13^{\circ}$ dia apresentassem números consideráveis de fêmeas ingurgitadas com este grau de infecção. $\mathrm{O}$ grau 4 foi registrado a partir do $12^{\circ}$ dia terminando no $17^{\circ}$ dia, com o pico máximo envolvendo $79,76 \%$ de fêmeas infectadas.

Nos resultados obtidos durante a fase experimental, verificou-se uma grande probabilidade em detectar a presença de esporocinetos de $\boldsymbol{B}$. bovis nas amostras coletadas durante o quinto dia de incubação, período em que $84 \%$ das fêmeas ingurgitadas evidenciaram esporocinetos na hemolinfa. Entretanto, a probabilidade em diagnosticar esta infecção, aumentou acentuadamente quando utilizado para exame amostras de hemolinfa coletadas com um período maior de incubação. Estas observações vêm reforçar a teoria sobre o efeito do período de incubação no grau de infecção das fêmeas ingurgitadas GAIDO \& GUGLIELMONE(1995), GUGLIELMONE etal.(1996, 1997) e MELENDEZ \& FORLANO (1996). Tais resultados divergem dos parâmetros encontrados por MAHONEY \& MIRRE (1971), que consideraram o período que corresponde entre o quinto e o sétimo dia de incubação como de pequena probabilidade para o diagnóstico de $\boldsymbol{B}$. bovis nas amostras de hemolinfa de fêmeas ingurgitadas de $\boldsymbol{B}$. microplus.

Ao contrário do que foi proposto por MAHONEY \& MIRRE (1971), AKINBOADE \& DIPEOLU (1981) descreverem a presença de esporocinetos de $B$. bovis durante o quinto e o $14^{\circ}$ dia de incubação das fêmeas ingurgitadas de Boophilus geigyi e consideraram o oitavo dia como ideal para o diagnóstico dos esporocinetos nas amostras de hemolinfa. QUINTÃO-SILVA \& RIBEIRO (2003), 
avaliando a taxa de infecção de fêmeas de $\boldsymbol{B}$. microplus em uma área de estabilidade enzoótica no Estado de Minas Gerais, Brasil, utilizaram o $8^{\circ}$ dia de incubação para o diagnóstico de Babesia spp. em amostras de hemolinfa. Em condições experimentais idênticas, GUGLIELMONE et al. (1985) observaram a presença de esporocinetos de $\boldsymbol{B}$. bovis em fêmeas ingurgitadas de $\boldsymbol{B}$. microplus durante período compreendido entre o quarto e o $10^{\circ}$ dia de incubação. Estes autores consideraram o nono e o $10^{\circ}$ dia de incubação como os ideais para diagnosticar a infecção das fêmeas ingurgitadas obtidas em bovinos com parasitemia de 1,5\% para $\boldsymbol{B}$. bigemina e $\boldsymbol{B}$. bovis e indicam que a falha no diagnóstico da infecção das fêmeas ingurgitadas de $\boldsymbol{B}$. microplus descritas por MAHONEY \& MIRRE (1971) está diretamente relacionada ao período inadequado em que as amostras de hemolinfa foram coletadas.

Das 84 fêmeas ingurgitadas comprovadamente infectadas, $100 \%$ das amostras de ovos apresentaram esporozoítos de $\boldsymbol{B}$. bovis, sendo $46,4 \%$ no quarto dia, $34,5 \%$ no quinto, $16,7 \%$ no sexto e $2,4 \%$ no sétimo dia pós-queda. A freqüência acumulada de $100 \%$ das amostras de ovos infectados foi evidenciada inicialmente no sétimo dia, permanecendo constante até o 17ํ dia pós-queda das fêmeas ingurgitadas.

A presença de esporocinetos de $\boldsymbol{B}$. bovis em amostras de ovos coletadas de fêmeas ingurgitadas, experimentalmente infectadas, também foi observada porCRAWLEY (1915), ROSENBUSCH(1927), DENNIS (1932), RIEK (1964,1966), AKINBOADE \& DIPEOLU (1981) eEL-ALLAWY (1977).

Durante a fase experimental, verificou-se um reduzido número de esporocinetos de $\boldsymbol{B}$. bovis, nas amostras de ovos coletadas entre o quarto e o sexto dia pós-queda das fêmeas ingurgitadas, entretanto, houve aumento acentuado quando as amostras foram obtidas a partir do sétimo dia. Estas observações permitiram caracterizar o efeito do tempo de incubação na probabilidade de detecção de esporocinetos nos ovos, bem como a realização da colheita e exame das amostras a partir do sétimo dia pós-queda das fêmeas ingurgitadas, aumentando assim a probabilidade do diagnóstico da infecção. Observações semelhantes foram encontradas por FARIAS (1994) ao estudar grupos de fêmeas ingurgitadas de $\boldsymbol{B}$. microplus infectados com amostras não modificadas de $\boldsymbol{B}$. bovis e B. bigemina

\section{CONCLUSÕES}

Os dados encontrados sobre a freqüência acumulada e o grau de infecção das fêmeas de $\boldsymbol{B}$. microplus infectadas nos permite afirmar que o período entre o quinto e o 17ํ dia de incubação é o indicado para obtenção das amostras de hemolinfa e o diagnóstico de $\boldsymbol{B}$. bovis no carrapato vetor. Após observar a freqüência acumulada de $100 \%$ das amostras de ovos infectados e o número crescente de esporocinetos neste período, concluiu-se que as amostras coletadas a partir do sétimo dia de incubação, permite o diagnóstico de $\boldsymbol{B}$. bovis mais preciso. O aumento do número de esporocinetos encontrados nas amostras de hemolinfa e ovos, durante a fase experimental, está relacionado ao período de incubação, e não ao grau de parasitemia do hospedeiro vertebrado.

\section{REFERÊNCIAS}

AKINBOADE, O.A.; DIPEOLU, O.O. Detection of Babesia bovis infections in Boophilus geigyi with egg crusinhgs larval smears, and haemolymph puncture. Veterinary Quartery, v.3, n.3, p.143-147, 1981.

BARREIRA, J.D. Caracterização morfológica, aspectos biológicos e patogenia das formas evolutivas de Babesia bigemina (Smith \& Kilborne, 1893) e Babesia bovis (Babés, 1888) (Protozoa: Babesiidae) em Boophilus microplus (Canestrini, 1887). 1988. 103f. Dissertação (Mestrado em Ciências Veterinárias) - Curso de Pós-graduação em Ciências Veterinárias. Universidade Federal Rural do Rio de Janeiro.

BARREIRA, J.D. Efeitos da infeç̧ão de Babesia bigemina (Smith \& Kilborne, 1893) e Babesia bovis (Babés, 1888) sobre os parâmetros biológicos do carrapato Boophilus microplus (Canestrini, 1887). 2001. 138f. Tese (Doutorado em Ciências Veterinárias) - Curso de Pós-graduação em Ciências Veterinárias, Universidade Federal Rural do Rio de Janeiro.

BENNETT, F.G. Oviposition of Boophilus microplus (Canestrini)(Acarida:Ixodidae). I. Influence of tick size on egg production. Acarologia, v.16, n.1, p.52-61, 1974.

BURGDORFER, W. A technique for detection of rickettsiae in tick. American Journal of Tropical Medicine and Hygiene, v.19, n.6, p.1010-1014, 1970.

CRAWLEY, H. Note on the stage of Piroplasma bigeminum wich occurs in the cattle tick, Margaropus annulatus. Journal Parasitology, v.2, n.1, p.87-92, 1915.

DE VOS, A.J. The effects of chemoterapy on Babesia bigemina in the tick vector Boophilus microplus. International Journal for Parasitology, v.14, n.2, p.249252, 1984.

DENNIS, E.W. The life cycle of Babesia bigemina (Smith and Kilborne) of Texas cattle fever in the tick, Margarapus annulatus (Say). University California. Publication in Zoology, v.36, n.2, p.263-298, 1932.

EL-ALLAWY, T.A. Detection of Piroplasma in the tick vector haemolymph and eggs smears of tick with special 
reference to the effect of incubation time on the infectivety of tick eggs. Agricultural Research Review, v.55, n.1, p.9-13, 1977

FARIAS, N.A.R. Efeito diferencial de Babesia bovis (Babés, 1888) e Babesia bigemina (Smith \& Kilborne, 1893) sobre a capacidade reprodutiva do vetor Boophilus microplus (Canestrini, 1887). 1994. 124f. Tese (Doutorado em Biologia Parasitária) - Fundação Oswaldo Cruz, RJ.

FRIEDHOFF, K.T. ; SMITH, R.D. Trasmission of Babesia of ticks. In: RISTIC, M. ; KREIER, J.P. Babesioses. New York : Academic, 1981. p.267-327.

GAIDO, A.B.; GUGLIELMONE, A.A. Infection dynamics of Babesia spp. Kinetes in naturally infected engorged female Boophilus microplus. Annals Tropical Medicine Parasitology, v.89, n.3, p.309-311, 1995.

GUGLIELMONE, A.A., da et al. Detection de merozoitos grandes (vermículos de Babesia em teleóginas de $\boldsymbol{B}$. microplus) alimentadas sobre terneros com distintos niveles de parasitemia de Babesia bigemina y Babesia bovis (B. argentina). Revista Iberica de Parasitología, v.46, n.3, p.303-311, 1985

GUGLIELMONE, A.A. da et al. Some quantitative aspect of natural babesial infection in the haemolymph of Boophilus microplus engorged female ticks. Parasitology, v.4, n.3, p.337-341, 1997.

GUGLIELMONE, A.A. et al. Light microscopy diagnosis of Babesia bovis and Babesia bigemina kinetes in the haemolymph of artificially infected Boophilus microplus engorged female ticks. Veterinary Parasitology, v.61, n.1, p.15-20, 1996.

KOCH, R. Beitrag zur entwicklungs geschichte der Piroplasmen. Zeistschrift fur Hygiene und Infektion, v.54, n.1, p.1-9, 1906

MAHONEY, D.F.; MIRRE, G.B. Bovine babesiasis; estimation of infection rates in the tick vector Boophilus microplus (Canestrini). Annal of Tropical Medicine and Parasitology, v.65, n.3, p.309-317, 1971.
MAHONEY, D.F.; MIRRE, G.B. The selection of larval of Boophilus microplus infected with Babesia bovis (Syn. B.argentina). Research Veterinary Science, v.23, n.1, p.126-127, 1977.

MELENDEZ, R.D.; FORLANO, M. Incidence and intensity of Babesia spp. Sporokinetes in engorged Boophilus microplus from a dairy herd in Venezuela. In: Vector Borne Pathogens International Trade and Tropical Animal Diseaes. New York : House of Uilemberge, 1996. p.148-156. 761p.

NEITZ, W.O. et al. Laboratory investigations on the life cycle on the Karoo paralysis tick (Ixodes rubicundus, Neumann, 1904). Onderstepoort Journal Veterinary Research, v.38, n.2, p.215-224, 1971.

QUINTÃO-SILVA, M.G.; RIBEIRO, M.F.B. Infection rate of Babesia sppl. Sporokinetes in engorged Boophilus microplus from na area of enzootic stability in the State of Minas Gerais, Brazil. Memórias Instituto Oswaldo Cruz, v.98, n.8, p.999-1002, 2003.

RIEK, R.F. The life cycle of Babesia bigemina (Smith \& Kilborne, 1983) in the tick vector Boophilus microplus. Australian Journal of Agriculture Research, v.15, n.8, p.802-821, 1964.

RIEK, R.F. The life cycle Babesia argentina (Lingnieris, 1903). (Sporozoa: Piroplasmidea) in the vector Boophilus microplus (Canestrini). Australian Journal Agriculture Research, v.17, n.2, p.247-254, 1966.

ROSENBUSCH, F. Estudies sobre la tristeza; evolution del Piroplasma bigemina en la garrapata (Boophilus microplus). Revista de la Universidad Buenos Aires, v.5, n.6, p.862-867, 1927.

SMITH, T.; KILBORNE, F.L. Investigation into the nature, causation, and prevention of Texas or Southern Cattle fever. Washington, DC : Bureau of animal Industry, U.S. Departmente of Agriculture, 1893. (Bulletin, n.1).

THOMPSON, K.C. A technique to stablish a laboratory colony of Boophilus microplus infected with Babesia bigemina. Veterinary Parasitology, v.2, n.2, p.223-229, 1976. 